This item was submitted to Loughborough's Research Repository by the author.

Items in Figshare are protected by copyright, with all rights reserved, unless otherwise indicated.

\title{
Smaller, lighter, faster? Reducing the carbon footprint of ambulances
}

PLEASE CITE THE PUBLISHED VERSION

https://doi.org/10.12968/jpar.2018.10.3.112

\section{PUBLISHER}

(C) MA Healthcare

\section{VERSION}

AM (Accepted Manuscript)

\section{PUBLISHER STATEMENT}

This work is made available according to the conditions of the Creative Commons Attribution-NonCommercialNoDerivatives 4.0 International (CC BY-NC-ND 4.0) licence. Full details of this licence are available at: https://creativecommons.org/licenses/by-nc-nd/4.0/

\section{LICENCE}

CC BY-NC-ND 4.0

\section{REPOSITORY RECORD}

Harmer, Luke, and Sue Hignett. 2019. "Smaller, Lighter, Faster? Reducing the Carbon Footprint of Ambulances". figshare. https://hdl.handle.net/2134/33095. 
Smaller, Lighter: Faster? Reducing the carbon footprint of ambulances.

Luke Harmer is a Lecturer at Loughborough University and Sue Hignett is Professor in Healthcare Ergonomics \& Patient Safety at Loughborough University.

Email for correspondence: L.A.Harmer@lboro.ac.uk

\section{Acknowledgements}

We would like to thank all of the respondents for their valuable contribution to this research and to the Niche Vehicle Network for their project support. 


\section{Smaller, Lighter: Faster? Reducing the carbon footprint of ambulances.}

\section{Key Points}

- Research on standardisation and modularisation has made a significant impact on dual crewed ambulance design for both clinical efficiency and patient experience. This knowledge needs to be transferred to solo response vehicles to optimise vehicle performance, clinical service provision and patient satisfaction.

- The project builds on previous Human Factors (Ergonomics) research in the FEVA, Future Ambulances, Smart Pods and CURE projects to improve clinical efficiency, staff and patient safety and patient experience.

- Ambulance Trusts have acknowledged the need to lower carbon emissions within the vehicle fleets but there are currently operational issues in using smaller vehicles

- New technologies and processes will allow for purpose-designed lighter weight and more fuel efficient vehicles.

- Smaller, lighter vehicles may become a more attractive choice as policy changes lead to a more integrated clinician service model

\section{Key Words}

-Ambulance design • Emergency medical services •Pre-hospital urgent care • Rapid Response Vehicles $\bullet$ Solo response technology $\bullet$ Carbon emissions

\section{Reflective questions}

In which cases could patients be prioritised and better served in primary care or community settings as opposed to transporting to ED's?

In the future what equipment, including vehicles, would enable clinicians to practise effectively to meet the needs of more patients outside of ED's?

When not transporting to ED's, what are the greatest safety challenges for patient treatment using emergency response vehicles?

How could clinical professionalism and trust be promoted further with patients not transported to ED's? 


\begin{abstract}
This paper outlines a feasibility project investigating the potential for smaller, lighter rapid response vehicles (RRVs) in reducing the carbon footprint and response times of ambulances.

Five stakeholder consultations were held with two Ambulance Trusts, an ambulance manufacturer, paramedic and the 'Ultra-Light Vehicle Group'1, to generate three novel design concepts for RRVs, which were then reviewed by four UK fleet managers and four clinicians.

The results indicated that the 'Integrated clinician service model' could create a future market for smaller, lighter vehicles. Reducing carbon emissions in the short term will most likely be achieved by; lower emission engines and improving engine and power management for Dual Crewed Ambulances. In the medium term (5-10 years) there will be a demand for low emission, composite light-weight Dual Crewed Ambulances.
\end{abstract}

\title{
Introduction
}

In order to meet the requirements for safer manual handling with equipment such as tail lifts and ramps Dual Crewed Ambulances (DCAs) are typically increasing in overall weight, resulting in reduced fuel economy, higher running costs and poorer driving characteristics. Additionally, the National Health Service (NHS) has identified a need for a more integrated clinical model (Department of Health, 2011) providing more 'see and treat' services instead of transporting patients to Emergency Departments. These two factors suggest that both evolved and entirely new types of emergency vehicles will be needed to meet future requirements of ambulance services.

This pilot project aimed to investigate the potential of smaller, lighter, Rapid Response Vehicles (RRVs) to:

- Reduce $\mathrm{CO}_{2}$ emissions (carbon footprint)

- Improve fuel efficiency

- Reduce the number of ambulance responses

- Reduce response times

- Increase operator safety (compared to motorcycles)

- Provide faster 'see and treat' services particularly in rural areas

\section{Vehicle Environmental Context}

The NHS accounts for 5\% of all road traffic in England, with staff, patients and visitors travelling over 20 billion kilometres annually by car (GrEAN, 2011). The Climate Change Act (2008) has committed the government to low carbon transport and Ambulance fleets have already considered low carbon solutions including an 'Eco Driving' initiative estimated to save $£ 1.5$ million per annum (GrEAN, 2011). Previous research at Loughborough

\footnotetext{
${ }^{1}$ The Ultra-Light Vehicle Group' is a research forum within the department of Automotive and Aeronautical Engineering at Loughborough University
} 
University considered the role of electric vehicles and highlighted the potential of solo response vehicles to reduce carbon emissions (Hignett et al, 2012).

\section{Vehicle Performance Context}

Changes in UK Government policy are encouraging a shift away from emergency care and towards urgent (pre-hospital) care by providing assessment, diagnosis and treatment for patients in their own homes or at the call site. These changes are increasing the number of solo responders with enhanced clinical skills (Department of Health, 2006).

Following the introduction of Ambulance Quality Indicators, Ambulance Services in England have been required to send an emergency response, with a defibrillator, within 8 minutes to 75\% of Category A (Red1) emergency calls (NEAS, 2017). Unfortunately, this standard has been frequently missed since its introduction in 2011 (NAO, 2017). After the emergency response had reached the scene it is reported that for over $50 \%$ of calls a dispatched ambulance was not required and the patient could have been referred to alternate medical treatment (BBC, 2014). About $40 \%$ of the 10.3 million visits to NHS emergency departments (EDs) in England in 2009/10 ended with the patient only needing advice and no specific treatment (HES online, 2010). It is reported that in some cases, such as heart attack and stroke, some patients have better outcomes by going straight to specialist centres but, for other patients, being seen in a primary or community care setting (i.e. not sent to ED) would provide more appropriate care and a better experience (NHS, 2013). This approach was explored by the CURE project, at Loughborough University (Hignett et al, 2012b), showing how non-critical, pre-hospital care could be delivered using portable pods /packs and mobile treatment units.

Other modes of transport have been trialled and found effective as RRVs. For example motorcycles in Scandinavia have been shown to respond on average 1 minute faster in cities (Nakstad et al, 2009). In the UK, the use of motorcycles has been limited and numbers reduced due to concerns about rider safety with respect to weather conditions and night working. However, a recent trial in Australia has illustrated their usefulness (EMS, 2015) providing "shorter response times for urgent cases and freed up road ambulances".

In 2015 the Association of Ambulance Chief Executives set out their vision of ambulance services for 2020 as an 'Integrated Services model' where the ambulance becomes a connected mobile treatment centre (AACE, 2015). This includes advanced clinicians who are trained and supported to manage more patients at home so that the ambulance sector becomes both a "coordinator of clinical and social responses and a mobile healthcare provider, operating in a range of settings".

\section{Phase 1: Stakeholder Consultation}

Method 
Five, 45 minute (approx.) stakeholder consultations were undertaken with two Ambulance Trust Fleet managers, the technical manager of a Vehicle Converter (ambulance manufacturer), , a paramedic with over 10years experience and the head of the Ultra-Light Vehicle Research Group to gain an understanding of current, short and medium term requirements with respect to:

- ambulance environmental impact and best practice

- advantages and disadvantages of specific vehicles

- impact of potential future working practices/ policy changes

- future design opportunities for new vehicles.

The semi-structured interview data were recorded and analysed qualitatively using a thematic approach resulting in 6 themes: vehicles used, responder working day, patient outcomes, future of service, reducing carbon emissions and ideal future vehicle.

\section{Results}

\section{Vehicles Used}

The majority of vehicles used by the two fleets were DCAs. Trust A had approximately 300 van conversion DCAs and Trust B had approximately 140 cab (box) conversions. Trust A expressed a preference for lowering the suspension (low floor kneeling) and ramp access to the ambulance because it was felt to be more reliable than the tail lift option. However, both Trusts reported that DCAs were not considered to generally have good handling characteristics.

Operationally, it was reported that the first response was usually with an RRV and followed by a DCA. The Trusts reported that $4 \times 4$ RRVs were preferred because of better driving characteristics in a range of conditions. Motorbike paramedics had not been used by the Trusts for a number of years because of concerns of rider safety, high vehicle running costs and limited operational conditions.

\section{Responder working day}

The responder working day was reported to be very varied including driving to standby points and waiting for the next call (with DCA staff sometimes waiting in the back of the vehicle between calls so that they are not visible to the public), lots of calls being stood down, significant waiting at EDs (Emergency Departments) between calls and very busy (full) shifts. Therefore, maintaining comfort and confidence of staff was felt to be key, including having the right kit, comfortable vehicle seating, heating and cooling; factors that can be influenced by both vehicle specification and manufacturer. There were reported to be increasing difficulties in recruiting staff for RRVs due to reluctance for lone working.

\section{Patient Outcomes}

The stakeholders reported that most calls do not necessarily require transporting but that patients are increasingly requesting to go to hospital as this is seen as the most risk adverse option and they (patients) believe that this will give a better outcome. However, in reality, they often have to wait longer than if they had booked an appointment with a GP or visited a walk-in centre. It was felt that patients generally were not aware of the differing types of emergency response and expected to receive an ambulance to a call. 


\section{Future of service}

Call response time targets were not felt to be a good measure of performance. The stakeholders expressed their opinion (and hope) that future targets should also include a measure of patient outcomes. They felt that in the longer term this would be more likely to encourage the 'at home' service options, which in turn would influence changes in vehicle types.

\section{Reducing Carbon Emissions}

The two Trusts reported that their fuel bills were approximately £6 million. It was discussed that targeting the right resources for the right jobs was a key method in reducing these costs, as well as not leaving vehicles on standby or being stood down (part way through a response). Alternative fuel vehicles (e.g. electric) were not seen as a workable solution in the short to medium term because of real world performance and operational compromises.

\section{Ideal Future Vehicles}

New technologies and processes are creating lightweight vehicles across the automotive industry and are becoming available for ambulance conversions. It was felt that there was the potential for the ambulance cab (box) conversions to move toward a complete composite construction to reduce overall weight. In particular there is an opportunity to consider specific design challenges such as creating a lightweight composite tail lift or ramp to reduce weight and improve driving characteristics.

It was also felt that there is an opportunity for smaller RRV vehicles, particularly within city centres but only in small numbers whilst DCA ambulances would continue to provide the majority of responses.

\section{Phase 2: Concept Generation and Evaluation}

The data from phase 1 was used to explore the new vehicle market potential by focussing on the apparent gaps (opportunities) to provide:

- Smaller lighter vehicles for first response

- New 'see and treat' vehicles for existing service provision and future integrated models.

These two opportunities were used to generate design concepts; Concept A for an agile fast response vehicle (cost range $£ 16,000-£ 35,000$ ) as an option between motorcycles and RRVs; and Concept B for a versatile RRV (cost range £35,000-£110,000) as an option between RRV and DCA vehicles.

\section{Concept A: Agile Fast Response Vehicle (FRV)}

Concept A proposed a smaller, lighter, agile FRV (Figure 1) to reach the patient quickly, particularly in congested urban areas or where access for a DCA would be difficult. This 
aimed to address duplication of resources where both an RRV and DCA were dispatched (with one being stood down during the response). For the future integrated care model with more patients treated at scene (home) the Agile FRV could provide a resource efficient method to reach patients. The vehicle would be single occupancy, fully enclosed, crash worthy and with good load carrying capacity whilst retaining the agility of a motorcycle.

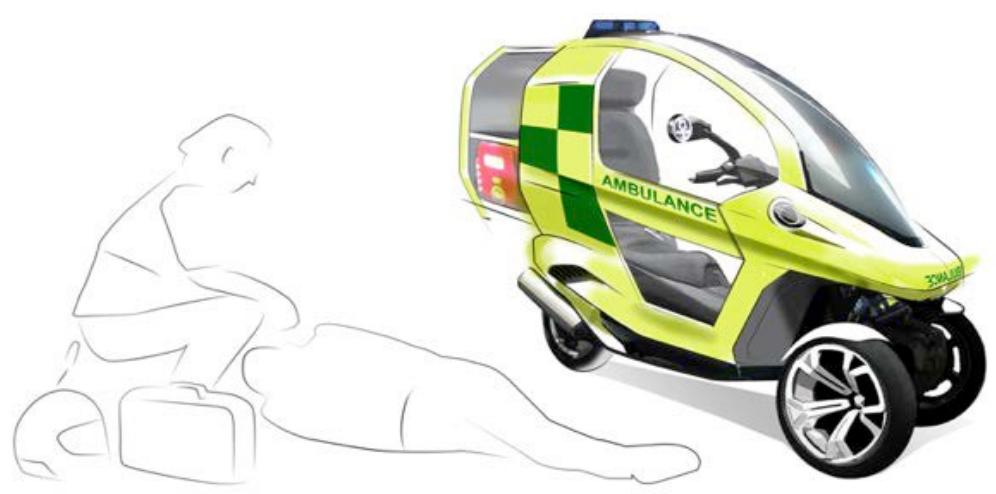

Figure 1: Agile FRV Concept

\section{Concept B: Versatile RRV with rear or side access}

This concept offered support for the future integrated service provision with a smaller vehicle than the DCA to replace current RRVs and support more options for treatment at scene. The design included the visibility of a DCA but with car-like driving characteristics. If the vehicle was dual staffed it could also be fitted for patient transport which might be particularly suitable for more mobile patients and those not requiring ED transportation. A key feature of the rear access vehicle (Figure 2) was the lowering floor to give easy level access and clearance to work whilst standing when the vehicle is stationary.

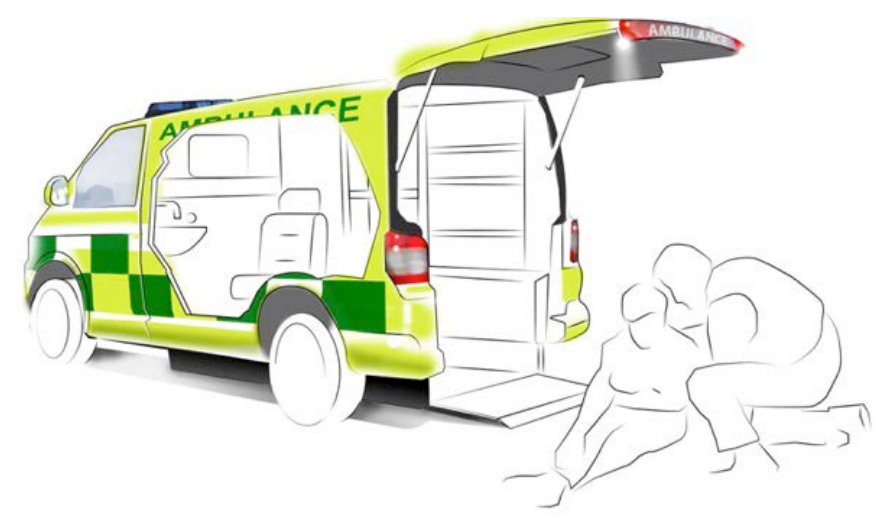

Figure 2. Concept B: Versatile RRV with rear access

The side access option (Figure 3) proposed a more cost effective design by using a rotating patient chair. It was proposed that this could require minimal vehicle modifications whilst still providing treatment capabilities within the vehicle. 


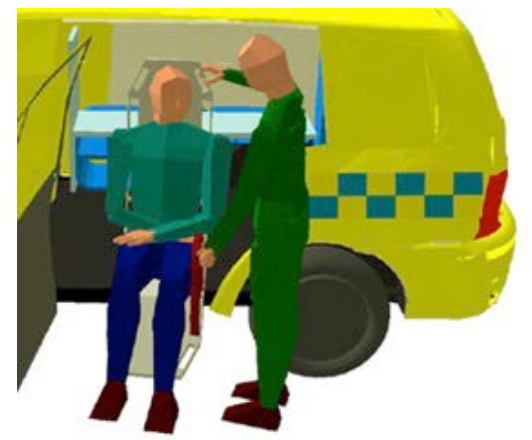

Figure 3: Concept B - Versatile RRV with side access, developed in SAMMIE CAD to investigate ergonomic requirements.

\section{Evaluation}

Evaluation of the concepts was invited using an online questionnaire with responses received from 4 NHS Trusts (total of 8 fleet managers and clinicians) across the UK. Two applicable patient scenarios (Table 1) developed by Hignett et al (2012b) were used to present the concepts and an adaptation of the traffic light model (RCA, 2010) was used to specify and score features using a five point Likert scale, enabling a comparison of the concepts with existing DCAs and RRVs for a scenario. The results of this vehicle feature comparison can be seen in Figure 4 and 5, the larger plot sizes illustrating higher respondent rating of feature suitability. Additional data were collected with telephone interviews to expand the responses where appropriate.

\begin{tabular}{|l|l|l|}
\hline Patient: & $\begin{array}{l}\text { Scenario 1 (RRV and Concept } \\
\text { A comparison) }\end{array}$ & $\begin{array}{l}\text { Scenario 2 (DCA and Concept } \\
\text { B comparison) }\end{array}$ \\
\hline Symptoms: & $\begin{array}{l}\text { Male, late 40's, known chronic } \\
\text { respiratory condition, Chest } \\
\text { pain. }\end{array}$ & Female, Elderly 70+ years. \\
\hline Shortness of breath on exertion. & $\begin{array}{l}\text { Open wound above left } \\
\text { eyebrow, significant bleeding } \\
\text { has stopped, some headache, } \\
\text { minor level of disorientation. }\end{array}$ \\
\hline History: & $\begin{array}{l}\text { Long term Chronic Obstructive } \\
\text { deterioration over 2 weeks. }\end{array}$ & $\begin{array}{l}\text { Mechanical fall, remembers } \\
\text { tripping over, hit head but } \\
\text { nothing specific, just ended up } \\
\text { on the floor. }\end{array}$ \\
\hline $\begin{array}{l}\text { Expected ECP } \\
\text { action: }\end{array}$ & $\begin{array}{l}\text { Full patient assessment, ECG } \\
\text { and respiratory assessment, }\end{array}$ & $\begin{array}{l}\text { Full patient assessment; blood } \\
\text { pressure, temp, blood sugar }\end{array}$ \\
\hline
\end{tabular}




\begin{tabular}{|l|l|l|}
\hline & nebulisers with oxygen therapy. & $\begin{array}{l}\text { monitoring, to establish reason } \\
\text { for fall. Two stitches above eye. }\end{array}$ \\
\hline Environment: & $\begin{array}{l}\text { Town centre, shopping district at } \\
\text { evening rush hour. }\end{array}$ & Rural village midday. \\
\hline
\end{tabular}

Table 1: Scenario's used to evaluate current vehicles and concepts

Replies

\section{Changes in Fleet Provision}

Three of the four Trusts had increased the number of DCAs over the last 15 years and envisioned that this trend would continue whilst RRV numbers were likely to decrease or remain the same.

"...the only resource we have added over the past 15/20 years are FRVs and this is only because they are faster and can hit the Cat As quicker. I can't see things changing much over the next 15 years; we need more DCAs to transport patients. We have tried educating the public not to call 999 but that has not worked".

Respondent 5 (Clinician)

\section{Reducing Carbon Emissions}

Respondents were asked to prioritise where they felt the greatest impact for reducing carbon emissions in the next 15 years could be achieved. Their ranked order list was:

1. More fuel efficient DCAs

2. Vehicles not being stood down

3. Smaller fuel efficient RRVs replacing DCA callouts

4. Better prediction of incident locations

5. Increase in 'see and treat' calls

6. Vehicles not left running on standby

7. Alternative fuelled vehicles

Additional interview details highlighted the impact on fuel consumption for vehicles left running on standby and on run-lock (where the vehicle is locked but left running to provide ancillary power). One Trust reported that almost $20 \%$ of vehicle running time was on runlock which did not include standby running with the crew inside the vehicle. This is perhaps an area where 'Stop-Start' technology has significant potential to improve functionality and fuel economy.

\section{Future Service Provision}

Ambulances will require improved patient assessment capacity to meet 'see-and-treat' service demands and will need to carry more sophisticated diagnostic and communication aids. 
"More 'see-and-treat' such as your small van concept with enhanced clinical skills for paramedics”. Respondent 8 (Clinician)

\section{Concept Feedback}

\section{Concept A: Agile RRV}

Half of the respondents felt that Concept A could be a useful addition to the fleet, but there were concerns about vehicle safety and crew satisfaction. However, the vehicle could provide a fast, cost effective response particularly in urban and congested areas.

“(Useful for) City centre work; busy events over large areas” Respondent 7 (Clinician)

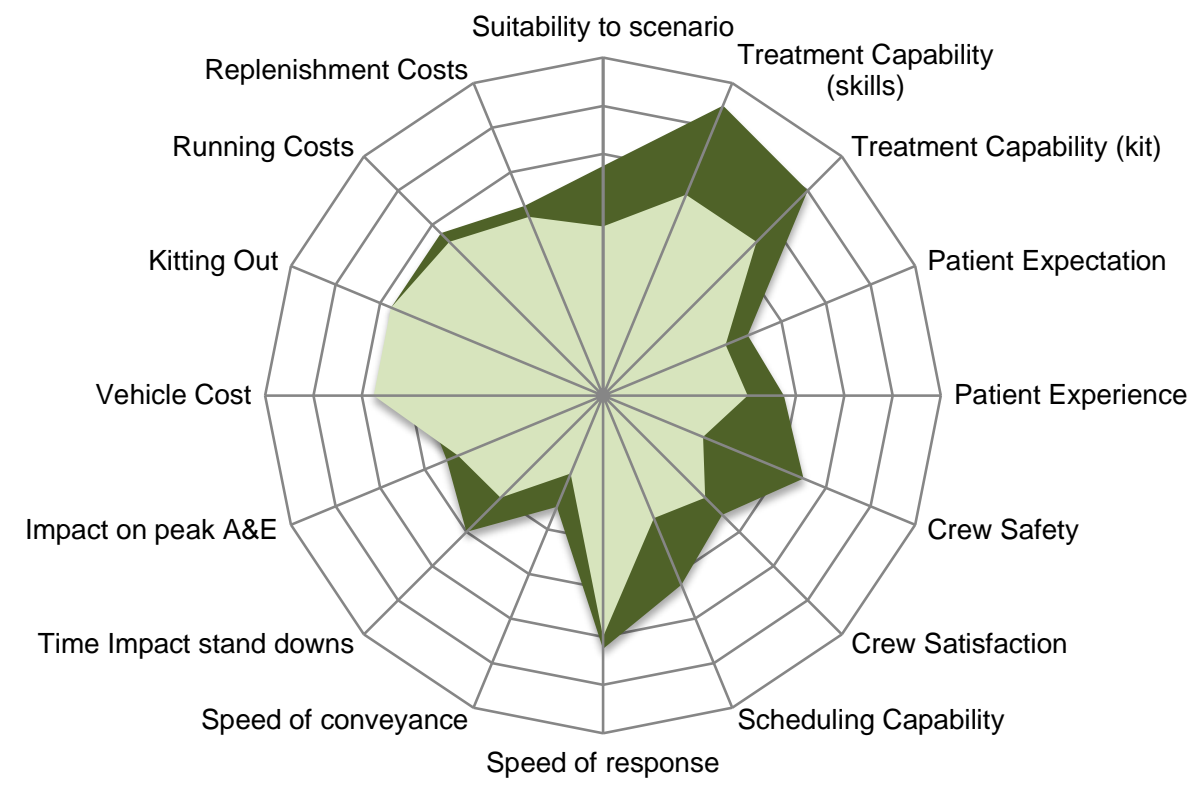

- Current RRV

Concept A

Figure 4: Respondent evaluation of current RRV and concept A to Scenario 1

\section{Concept B: Versatile RRV rear/side access}

Concept B was also felt to offer some design options as a useful addition to the fleet. Concerns were raised by Fleet Managers that for the cost outlay of the new design it might not provide significant advantages over a DCA (van conversion) and that there may be reliability issues with the lowering floor. Clinicians were generally more positive about Concept B over concept A.

"(Useful for) any RRV scenario, good opportunity to get patients out of the public eye. Very worthwhile and better than traditional $R R V$ ” Respondent 5 (Clinician) 


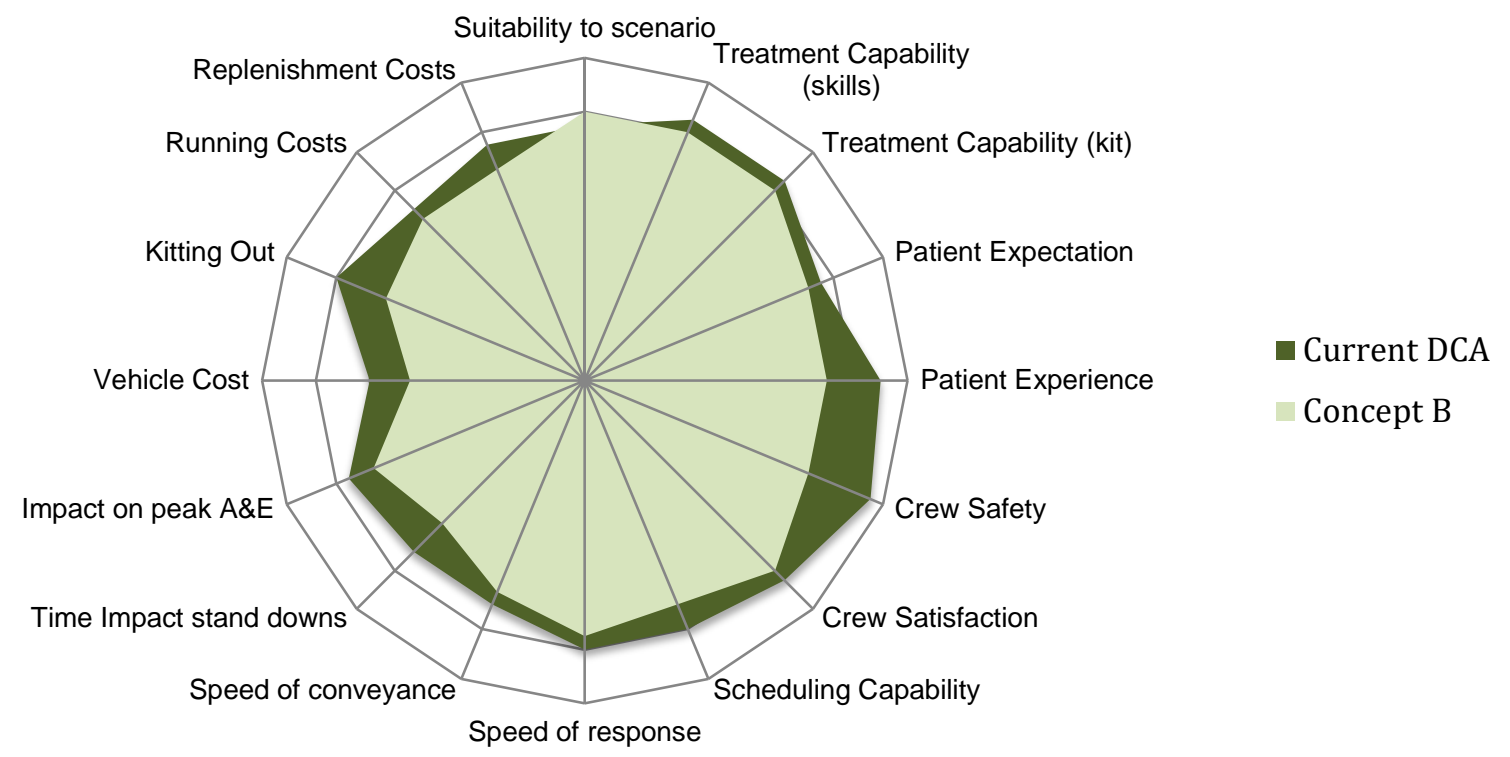

Figure 5: Respondent evaluation of current DCA and concept B to Scenario 2

The side access option for Concept B was felt to be useful for getting patients into a vehicle but there were concerns that if a patient required treatment laying down this could be complex and that clinical infection control could be harder to manage in the smaller space provided.

\section{Concept C: Superlight DCA}

During the evaluation phase, it emerged that in the short/medium term a superlight DCA (Concept $\mathrm{C}$ ) could provide the greatest benefit and allow for flexibility in service provision Reducing vehicle weight could be best addressed through cost-focussed design innovation (NVN, 2015). For the van conversion utilising composites to address weight issues such as tail lifts and equipment storage through an integrated vehicle structure.

\section{Conclusion and Further Work}

This feasibility study indicates that policy change to support the integrated clinician service model could create a future market for smaller, lighter vehicles. The study indicates that the most pressing issue is to improve fuel economy in the immediate future and this would probably be best achieved through increased fuel economy via the adoption of technological solutions for existing DCAs. 
Three new vehicle types have been identified to inform further research. There is a small potential market for an agile single crew FRV particularly in urban areas. This type of vehicle could be based on an adaptation of a three-wheeled leaning scooter, but would require development of passive safety and car-like crash testing to demonstrate suitability. The demand for these vehicles is estimated to be up to 20 vehicles per Trust.

There is a small initial market (up to 100 per Trust) for a versatile RRV that is likely to increase as the Ambulance service moves toward an integrated service provision with more 'see-and-treat' cases

However, the feasibility study indicated that the superlight DCA might have the greatest market potential in the short to medium term (up to 500 per Trust). As cities require ultra low emission vehicles and if a comparable cost can be achieved, there are opportunities to minimise the weight of DCAs using composites (e.g. monocoque for the vehicle structure, internal and external panels) to reduce carbon emissions and improve driving performance.

Further work across all the UK trusts should be undertaken to enable a more detailed picture of future vehicle demand. Additionally a study specifically designed to research patient perceptions of using non-DCA vehicles (when clinically appropriate to a scenario) will help understand the role of vehicles in supporting an integrated clinician service model to enable "care as close to home as possible" (House of Commons, 2017).

\section{References}

Association of Ambulance Chief Executives (2015) A vision for the ambulance service: '2020 and beyond' and the steps to its realisation. http://aace.org.uk/wpcontent/uploads/2015/09/Ambulance-2020-and-beyond-the-AACE-vision.pdf (Accessed 19th September 2017)

BBC. (2014). Ambulance targets: Plan for longer times in England revealed. BBC News: http://www.bbc.co.uk/news/uk-30566207 (Accessed 20th October 2015)

Climate Change Act. (2008). https://www.legislation.gov.uk/ukpga/2008/27/contents (Accessed 19th September 2017)

Department of Health. (2006). Direction of travel for urgent care: A discussion document. London: Department of Health.

Department of Health. (2011). Transforming NHS ambulance services. London: National Audit Office.

EMS. (2015). Motorcycle medics prove useful. http://www.ems1.com/paramedicchief/articles/13540048-Motorcycle-medics-prove-useful/ (Accessed 9th January 2016)

GrEAN Green Environmental Ambulance Network. (2011). Green Passport. http://aace.org.uk/wp-content/uploads/2012/03/GrEAN_passport_large.pdf (Accessed 19 Sept. 2017) 
HESonline (2010) ED Data (experimental statistics) 2009-10 www.hesonline.nhs.uk/Ease/servlet/ContentServer?siteI

House of Commons. (2017) NHS ambulance services: Sixty-second Report of Session 2016-17 https://publications.parliament.uk/pa/cm201617/cmselect/cmpubacc/1035/1035.pdf

Hignett, S., Baker, W., Storer, I., \& Mansfield, N. (2012a). Future electric vehicles for ambulances (FEVA). Journal of Paramedic Practice, 77-82.

Hignett, S., Fray, M., \& Benger, J. (2012b). CURE (Community Urgent Response Environment) Portable Work Stations. Journal of Paramedic Practice, 352-358.

Nakstad, A. B. (2009). Medical emergency motorcycle - is it useful in a Scandinavian Emergency Medical Service? Scandinavian Journal of Trauma Resuscitation \& Emergency Medicine. 17; 9-12

NAO, (2017). NHS Ambulance Services. https://www.nao.org.uk/wpcontent/uploads/2017/01/NHS-Ambulance-Services-Summary.pdf (Accessed 19th Sept 2017)

NEAS, (2017) Ambulance response categories explained, https://www.neas.nhs.uk/ourservices/accident-emergency/ambulance-response-categories-explained.aspx (Accessed 19th Sept 2017)

NHS England. (2013). Urgent and Emergency Care Review - Evidence Base Engagement. NHS. https://www.england.nhs.uk/wp-content/uploads/2013/06/urg-emerg-care-ev-bse.pdf

Oughtred and Harrison Facilities Ltd, J \& A Kay Ltd, Panel Systems. (2015). The Development and Testing of a Lightweight ED Ambulance. Loughborough: Niche Vehicle Network. Internal Report.

RCA. (2009) Health care on the move, Treating Patients in the Community: The Smart Pods Project http://www.hhc.rca.ac.uk/CMS/files/HealthcareOnTheMove-lowres.pdf (Accessed 19th Sept 2017)

Yorkshire Ambulance Service (2017) Accident and Emergency. http://www.yas.nhs.uk/OurServices/a accidentandemergency.html. (Accessed 19th Sept 2017) 